The role of linguistic experience in the hemispheric processing of lexical tone

\author{
by Yue Wang \\ Dawn Behne \\ Allard Jongman \\ and Joan A Sereno
}

2004

This is the published version of the article, made available with the permission of the publisher. The original published version can be found at the link below.

Wang, Y., Behne, D., Jongman, A., and Sereno, J. The role of linguistic experience in the hemispheric processing of lexical tone. Applied Psycholinguistics 25 (2004), 449-466.

Published version: http://dx.doi.org/10.1017/So142716404001213

Terms of Use: http://www2.ku.edu/ scholar/docs/license.shtml

KU ScholarWorks is a service provided by the KU Libraries' Office of Scholarly Communication \& Copyright. 
Applied Psycholinguistics 25 (2004), 449-466

Printed in the United States of America

DOI: 10.1017.S0142716404001213

\title{
The role of linguistic experience in the hemispheric processing of lexical tone
}

\author{
YUE WANG \\ Simon Fraser University \\ DAWN M. BEHNE \\ Norwegian University of Science and Technology
}

ALLARD JONGMAN and JOAN A. SERENO

University of Kansas

ADDRESS FOR CORRESPONDENCE

Yue Wang, Department of Linguistics, Simon Fraser University, 8301 Robert C. Brown Building, Burnaby, BC, Canada, V5A 1S6. E-mail: yuew@sfu.ca

ABSTRACT

This study investigated hemispheric lateralization of Mandarin tone. Four groups of listeners were examined: native Mandarin listeners, English-Mandarin bilinguals, Norwegian listeners with experience with Norwegian tone, and American listeners with no tone experience. Tone pairs were dichotically presented and listeners identified which tone they heard in each ear. For the Mandarin listeners, $57 \%$ of the total errors occurred in the left ear, indicating a right-ear (left-hemisphere) advantage. The EnglishMandarin bilinguals exhibited nativelike patterns, with 56\% left-ear errors. However, no ear advantage was found for the Norwegian or American listeners (48 and 47\% left-ear errors, respectively). Results indicate left-hemisphere dominance of Mandarin tone by native and proficient bilingual listeners, whereas nonnative listeners show no evidence of lateralization, regardless of their familiarity with lexical tone.

Although the left hemisphere is more involved in processing analytic linguistic information such as phonemes and words (e.g., Kimura, 1964; Shankweiler \& Studdert-Kennedy, 1967; Studdert-Kennedy \& Shankweiler, 1970), pitch processing occurs primarily in the right hemisphere (Curry, 1967; Goodglass \& Calderon, 1977; Kimura, 1964; Mazzucchi, Parma, \& Cattelani, 1981; Zatorre, Evans, Meyer, \& Gjedde, 1992). The lateralization of lexical tone, where pitch is used to distinguish word meaning, provides a unique case. On the one hand, tones involve a modulation of pitch, generally found to be the domain of the right hemisphere; on the other hand, they are used to make linguistic contrasts, assumed to be a function of the left hemisphere (Ryalls \& Reinvang, 1986). The processing of lexical tone is thus related to two competing hypotheses with regard to the nature of the mechanisms underlying hemispheric specialization of pitch: the acoustic

(C) 2004 Cambridge University Press 0142-7164/04 \$12.00 
(or cue-dependent) hypothesis, and the functional (or task-dependent) hypothesis (Gandour et al., 2003; Wong, 2002). The acoustic hypothesis posits that lateralization of processing depends on acoustic properties (e.g., Robin, Tranel, \& Damasio, 1990), indicating that lexical tone is processed within the scope of general pitch processing, which is lateralized in the right hemisphere. The functional hypothesis, on the other hand, proposes that lateralization of processing is functionally determined, depending on whether pitch is used to convey distinctive linguistic information (e.g., Van Lancker, 1980), inferring a left-hemisphere specification of lexical tone as a linguistic property.

To investigate lateralization of lexical tone in light of these two hypotheses, it is necessary to include not only native speakers of a tone language but also native speakers of languages with either different tonal contrasts or no tone contrasts at all, to determine if and to what extent lexical tone is processed as distinctive linguistic information. The present study investigated the lateralization of Mandarin Chinese lexical tones by native speakers of Mandarin; English-Mandarin bilinguals who are highly proficient in Mandarin; native speakers of Norwegian, whose native language has tonal distinctions; and native speakers of American English with no prior experience of lexical tone.

Every Mandarin syllable carries a tone. Mandarin phonemically distinguishes four tones differing primarily in pitch height and contour shape, with Tone 1 having high-level pitch, Tone 2 mid-rising pitch, Tone 3 low-dipping pitch, and Tone 4 high-falling pitch. In Norwegian, two distinctive pitch patterns are used for lexical contrasts in most dialects. Unlike Mandarin, Norwegian tonal contrasts occur between words with more than one syllable where the two distinctive tones ${ }^{1}$ are realized only on a stressed syllable when it is followed by one or more unstressed syllables (Fintoft, 1970; Kristoffersen, 2000).

To investigate lateralization of tone, a dichotic listening task, involving the simultaneous presentation of a pair of different stimuli to the right and left ear, was used. Research using dichotic presentation has regularly shown a right ear advantage (i.e., a left hemisphere dominance) for linguistic stimuli (e.g., Bryden \& Murray, 1985; Cutting, 1974; Dwyer, Blumstein, \& Ryalls, 1982), attributable to the right ear being more directly connected to the language-dominant left hemisphere and the left ear to the nondominant right hemisphere through the primary contralateral pathways (e.g., Kimura, 1967). More specifically, for perception of lexical tone by native speakers, previous research using the dichotic paradigm has consistently found a right ear advantage (REA) for tone processing. An REA generally occurs in the processing of the tonal distinctions in Thai (Van Lancker \& Fromkin, 1973), Norwegian (Moen, 1993), and Mandarin Chinese (Wang, Jongman, \& Sereno, 2001) by native speakers of these languages. However, when native speakers of a tone language (Thai) were tested on hummed tones (i.e., pitch changes alone), no ear advantage was found (Van Lancker \& Fromkin, 1973). Similarly, no ear advantage was found for native speakers of a language without tonal contrasts, such as American English when processing Thai (Van Lacker \& Fromkin, 1973) or Mandarin (Wang et al., 2001) tones, even when the nonnative speakers were musically trained (Van Lancker and Fromkin, 1978).

Consistent with the dichotic testing results, studies of aphasic speakers indicate that for Mandarin (Naeser \& Chan, 1980; Packard, 1986), Thai (Gandour \& 
Dardarananda, 1983; Gandour, Petty, \& Dardarananda, 1988), and Norwegian (Ryalls \& Reinvang, 1986) aphasics, damage to the left hemisphere (rather than to the right hemisphere) impairs both tone production and perception, indicating a left-hemisphere dominance for lexical tone. Recent studies using neuroimaging techniques such as functional magnetic resonance imaging (fMRI) and positron emission tomography (PET) have also demonstrated that processing of Mandarin (Klein, Zatorrre, Milner, \& Zhao, 2001; Wang, Sereno, Jongman, \& Hirsch, 2003) and Thai (Gandour, Wong, Hsieh, Weinzapfel, Van Lancker, \& Hutchins, 2000) tones by native speakers of these languages predominantly involves the left-hemisphere functional regions, whereas this hemispheric specification is not characteristic of nonnative speakers (Wang et al., 2003).

These results consistently show that native speakers of tone languages process tonal contrasts predominantly in the left hemisphere, whereas linguistically irrelevant pitch information such as hums yielded no significant ear effects. For nonnative speakers whose native languages do not have tonal distinctions, tones as well as hums were not processed as language and were thus not lateralized in the left hemisphere. Taken together, these studies suggest that left-hemisphere specialization for tones occurs only when they are part of the speaker's linguistic system.

Two subsequent questions arise given the above-mentioned patterns of tone processing by native and naive speakers. First, what is the hemispheric processing pattern for naive speakers of a tone language whose native language also has tonal distinctions? A PET study of Thai tones (Gandour et al., 2000) shows that, for native speakers of Chinese with no Thai background, Thai tones were not processed in left-hemisphere language-sensitive regions, as was exhibited by native Thai speakers. However, there are few behavioral studies to confirm this. In the present study, native listeners of Norwegian are included in the examination of the processing of Mandarin tones, since Norwegian has tonal distinctions that are processed primarily in the left hemisphere (Moen, 1993).

A second question concerns the hemispheric specialization of bilingual speakers who have been exposed to a tone language as a second language (L2). Lateralization of language processing by bilinguals and L2 learners has been controversial in the literature. Some studies show that the languages of bilinguals are left lateralized to the same extent as for monolinguals (e.g., Soares, 1982, 1984; Soares \& Grosjean, 1981), whereas others demonstrate that bilingual processing is mainly a left-hemisphere function, but with greater right hemisphere involvement than for monolinguals (e.g., Mildner, 1999). Still others show an interhemispheric processing (equal processing in both hemispheres) in bilinguals (Ke, 1992). In studies that systematically manipulated age of L2 acquisition, Sussman, Franklin, and Simon (1982) demonstrated left-hemisphere dominance for early bilinguals and symmetrical hemisphere involvement for late bilinguals; and Wuillemin and Richardson (1994) found greater left hemisphere involvement for early bilinguals and greater right hemisphere involvement for late bilinguals. With respect to proficiency, Albanese (1985) found that growing L2 proficiency increases right hemisphere participation, but in Schouten et al. (1985), both proficient and nonproficient bilinguals show left hemisphere dominance for the $\mathrm{L} 2$. These inconsistent 
findings suggest that caution should be taken when comparing and interpreting the processing patterns of bilinguals, given that listeners' linguistic experience was not always comparable across these studies. Although there are few studies on the bilingual processing of Mandarin tone, fMRI research has observed cortical modification in the process of learning Mandarin tone by elementary nonnative learners (Wang et al., 2003), with increased activation in left hemisphere language-related areas and recruitment of right hemispheric areas as a function of L2 proficiency. The present study includes a group of highly proficient early English-Mandarin bilinguals, representing speakers with ultimate attainment of Mandarin.

In sum, the present study investigates the processing of Mandarin tones by four groups of listeners with different experience with tone: on the one extreme are the Chinese speakers for whom Mandarin tone is native, on the other extreme are the American English speakers without any tone language background; and in between are the Chinese-English bilinguals who have achieved ultimate proficiency in Mandarin and Norwegian speakers with tone experience in their native language. These four groups of speakers allow us to provide a relatively broad profile of the patterns of hemispheric specialization in the processing of Mandarin tones by native and nonnative speakers.

\section{METHOD}

\section{Participants}

The participants include 20 native speakers of Mandarin Chinese (11 females, 9 males), 15 English-Mandarin bilingual speakers (9 females, 6 males), 20 native speakers of Norwegian (12 females, 8 males), and 20 native speakers of American English (11 females, 9 males ). ${ }^{2}$ They were all adult listeners with no known history of speech and hearing impairments, and all were right-handed according to an assessment with the Edinburgh Handedness Inventory (Oldfield, 1971). The Chinese, bilingual, and American participants were all students at Cornell University, and the Norwegian participants were students at the Norwegian University of Science and Technology.

The Chinese listeners all had some previous exposure to English, with 8 years' class instruction in China and less than 6 years of residence in the United States. The English-Mandarin bilingual speakers were born and raised in the United States; all claimed to have acquired English as their first and dominant language. Since they all had native Chinese parents or family members, they had been exposed to Mandarin (speaking and/or comprehension) before and since school age. They also had received formal class instruction in Mandarin Chinese for 2-4 years in secondary school and for 2-3 years at Cornell University. They were therefore fluent in Mandarin as well as English. The Norwegian participants had no knowledge of Mandarin Chinese prior to the present experiment. However, they were all native speakers of a Norwegian dialect that has tonal distinctions. The American participants had no prior knowledge of Mandarin Chinese or any other tonal languages. 
The stimuli were 16 commonly used monosyllabic Mandarin words, consisting of four different syllables (fan, guo, hui, shi), each combined with the four tones, resulting in four tone quadruplets (see Appendix A for the stimuli and glosses).

The stimuli were produced by a female native speaker of Mandarin Chinese, who was recorded in a sound-treated booth in the Cornell Phonetics Laboratory, using a cardioid microphone (Electrovoice RE 20) and a cassette recorder (Carver TD1700). The sixteen target words were produced in isolation. Twenty repetitions of each target word were produced at a variety of speaking rates. The recordings were then digitized at $11.025 \mathrm{kHz}$ after low-pass filtering at $5 \mathrm{kHz}$, using WAVES+/ESPS speech analysis software running on a SUN SPARCstation.

In order to create dichotic pairs that were matched for duration and intensity, tokens were selected and edited from among the recorded target words in two different ways. First, among the 20 repetitions of each word, the tone quadruplets for each syllable were selected such that their durational difference was under $10 \%$, the approximate just noticeable difference for duration (Lehiste, 1970). Second, the intensity of the 16 selected words was equalized such that the root mean square amplitude of all the resulting stimuli was the same. The mean duration of the stimuli was $437 \mathrm{~ms}$, and the amplitude was $75 \mathrm{~dB}$.

A pilot study with four Chinese listeners using dichotic stimuli showed a low (5\%) error rate on average. To induce errors, the target stimuli were each embedded in Gaussian noise with the same duration as the corresponding stimulus. The stimuli for the bilingual, Norwegian, and American listeners were also embedded in noise in the same fashion. However, to generate error rates that were comparable across the four listener groups, the appropriate signal to noise $(\mathrm{S} / \mathrm{N})$ ratio, as well as interstimulus interval (ISI), were established empirically by testing two listeners in each group to achieve a mean error rate of approximately $34 \%$ across pilot listeners (range $=31-37 \%$ ). This resulted in a $\mathrm{S} / \mathrm{N}$ ratio of $-10 \mathrm{~dB}$ for the Chinese and bilingual listeners, and $0 \mathrm{~dB}$ for the Norwegian and American listeners; and an ISI of $2 \mathrm{~ms}$ for the Chinese, bilingual, and Norwegian listeners and $4 \mathrm{~ms}$ for the American listeners.

These tokens were then transferred to a PC for pairing and presentation of the dichotic stimuli using the BLISS system (Mertus, 1989). A total of 48 dichotic pairs (12 pairs $\times 4$ syllables) were generated with all possible pairings for each of the four syllables, with the exception of identical pairings. The segmental composition was always the same within each pair. These 48 dichotic pairs were repeated four times, resulting in a total of 192 pairs for the dichotic test.

\section{Procedure}

For the Chinese, bilingual, and American listeners, the experiment was conducted in the Cornell Phonetics Laboratory, and for the Norwegian listeners, in the Phonetics Laboratory of the Norwegian University of Science and Technology. All listeners were tested individually over Sony MDR-V6 headphones.

Prior to the dichotic test, the Chinese and bilingual listeners were submitted to a pretest with the 16 target stimuli presented binaurally without noise in a random order. The listeners in this study all met the criterion of perfect identification of the 
four tones. The Norwegian and American listeners received a short training program (about $30 \mathrm{~min}$ ) before the pretest, to familiarize them with the four Mandarin tones. They were only presented with the 16 target stimuli in isolation, produced by the same speaker as in the test. No attempt was made to have them associate these stimuli with any meaning. Only listeners who could identify $75 \%$ or more of the tones correctly in the pretest were retained in the dichotic test. Two Norwegian and three American listeners did not reach this criterion.

The dichotic test contained four randomized blocks (i.e., four repetitions) of 48 dichotic pairs each. For each listener, the output volume of the two channels of the headphones was calibrated with a sound level meter such that it was always $75 \mathrm{~dB}$ for both channels. Thus, equal intensity was maintained for both ears.

Listeners were instructed that they would be hearing two different tones (with the same segmental components) simultaneously over the headphones: one in the right ear and the other in the left ear. They were to identify both stimuli on an answer sheet. Right ear and left ear response rows (each with four numerical tone marks) were counterbalanced across blocks to avoid order bias. In addition, to eliminate channel effects, the headphones were reversed after two blocks. Headphone channels were counterbalanced across listeners.

The instructions and answer sheets were presented in Chinese to the Chinese listeners, in English to the bilingual and American listeners, and in Norwegian to the Norwegian listeners. Only one listener was tested at a time. The test lasted approximately $30 \mathrm{~min}$ for the Chinese and bilingual listeners, whereas the test and training lasted approximately $60 \mathrm{~min}$ for the Norwegian and American listeners. To avoid fatigue, these listeners took a short break between training and the dichotic test.

\section{Data analysis}

The number of errors in each ear was tallied. Two measures were calculated for each listener: the difference in the number of errors made for the left and right ears and the percentage of errors $(\mathrm{POE})$, defined as $\left[P_{\mathrm{L}} /\left(P_{\mathrm{R}}+P_{\mathrm{L}}\right)\right] \times 100$, where $P_{\mathrm{L}}$ is percentage errors in the left ear and $P_{\mathrm{R}}$ is percentage errors in the right ear. A POE value of $60 \%$ therefore means the left ear makes $60 \%$ of the total errors. The index ranges from $0 \%$ for perfect left ear advantage (LEA), to $50 \%$ for no advantage for either ear, to $100 \%$ for perfect right ear advantage (REA), thus indicating the degree of laterality. (For the rationale on using these measures, see Wang et al., 2001.)

When calculating the number of errors and POE, both-ear errors in a dichotic pair (i.e., neither tone is identified correctly) were not included, because previous research indicated that only those trials in which listeners identify one item correctly, and fail to identify the other, provide accurate information about laterality (Bryden, 1988).

\section{RESULTS}

Overall, out of a total of 384 stimuli (192 pairs), the error rate is $45 \%$ for the Chinese listeners, $46 \%$ for the bilinguals, $49 \%$ for the Norwegian listeners, and $46 \%$ for 
Wang et al.: Hemispheric processing of lexical tone

Table 1. Mean number of correct and incorrect responses for the 192 pairs of stimuli for the four groups of listeners (Chinese, English-Mandarin bilinguals, Norwegian, and American)

\begin{tabular}{lcccc}
\hline \hline & LX-RC & LC-RX & LX-RX & LC-RC \\
\hline Chinese & 39 & 31 & 52 & 70 \\
Bilingual & 41 & 33 & 51 & 67 \\
Norwegian & 32 & 35 & 60 & 65 \\
American & 34 & 37 & 53 & 68 \\
\hline \hline
\end{tabular}

Note: LX, left ear wrong; LC, left ear correct; RX, right ear wrong; $\mathrm{RC}$, right ear correct.

the American listeners. The similarity of error rates for the four groups of listeners suggests that the manipulation of the $\mathrm{S} / \mathrm{N}$ ratio and ISI was highly successful in matching error rates and therefore justifies comparison across groups.

Table 1 lists the number of correct and incorrect responses for each group; that is, the number of left-ear errors, right-ear errors, both-ear errors, and both-ear correct responses.

\section{Percentage of errors}

Figure 1 displays the listeners' performance in terms of the percentage of left and right ear errors, showing that the POE of the four groups are 57\% (Chinese), 56\% (bilingual), 47\% (Norwegian), and 48\% (American).

These data were analyzed using a one-factor analysis of variance (ANOVA) with POE as the dependent variable, and listener group (Chinese, Bilingual, Norwegian, American) as the independent variable. As expected, there was a significant main effect of listener group, $F(3,71)=5.7, p<.002$. Post hoc analysis (Tukey honestly significant difference [HSD]) showed that the Chinese and bilingual groups were significantly different from the Norwegian and American groups in ear preference. For the Chinese and bilingual listeners, the percentage of errors for the left ear exceeds that for the right ear, indicating an REA. In contrast, for the Norwegian and American listeners, errors for the left and right ears are comparable, showing little ear difference.

\section{Frequency of REA occurrence}

Figure 2 shows the number of individuals showing an REA in each listener group, Chinese, bilingual, Norwegian, and American. As shown in the figure, for the Chinese listeners, 15 out of 20 listeners made more errors on the left than on the right ear, indicating an REA, whereas the remaining five listeners exhibited a tendency toward an LEA. This difference in the number of Chinese listeners showing an ear advantage was significant, $\chi^{2}(1)=5, p<.025$, indicating greater frequency of occurrence of an REA than an LEA. Similar to the native Chinese patterns, 12 out of 15 bilingual listeners exhibited an REA, $\chi^{2}(1)=5.4, p<.002$. In contrast, for the Norwegian group, although 7 out of 20 listeners had more 
Wang et al.: Hemispheric processing of lexical tone

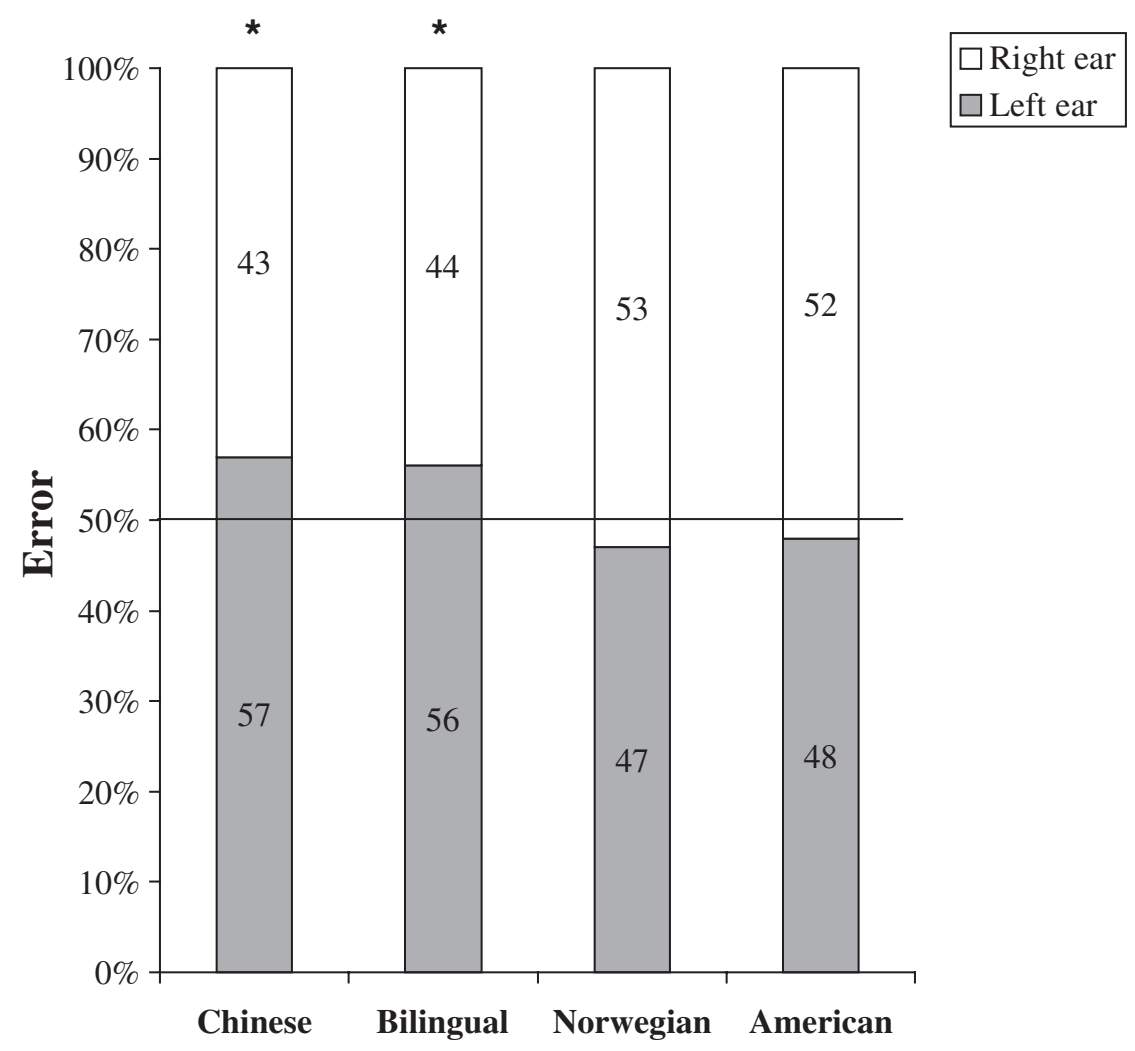

Figure 1. The distribution of left-ear errors and right-ear errors $(\%)$ for the Chinese $(n=20)$, bilingual $(n=15)$, Norwegian $(n=20)$, and American $(n=20)$ listeners. ${ }^{*}$ The difference in right-ear and left-ear errors is significant at $p<.05$.

left-ear than right-ear errors, the number of listeners with an ear preference was not significantly different, $\chi^{2}(1)=1.8, p>.180$. Likewise, for the American group, the number of listeners showing an ear preference was not significantly different, $\chi^{2}(1)=0.8, p>.371$, with 8 out of 20 listeners revealing a slight tendency toward an REA. These patterns are consistent with the POE results, showing greater frequency of an REA for the Chinese and bilingual listeners but no ear preference for the Norwegian and American listeners.

\section{Individual tones}

Table 2 presents the mean number of errors occurring in the left and right ear for each tone. Consistent with the overall result showing an REA, for the individual tones the mean number of left-ear errors is greater than that of right-ear errors for the Chinese and bilingual listeners. No such pattern was exhibited for the 
Wang et al.: Hemispheric processing of lexical tone

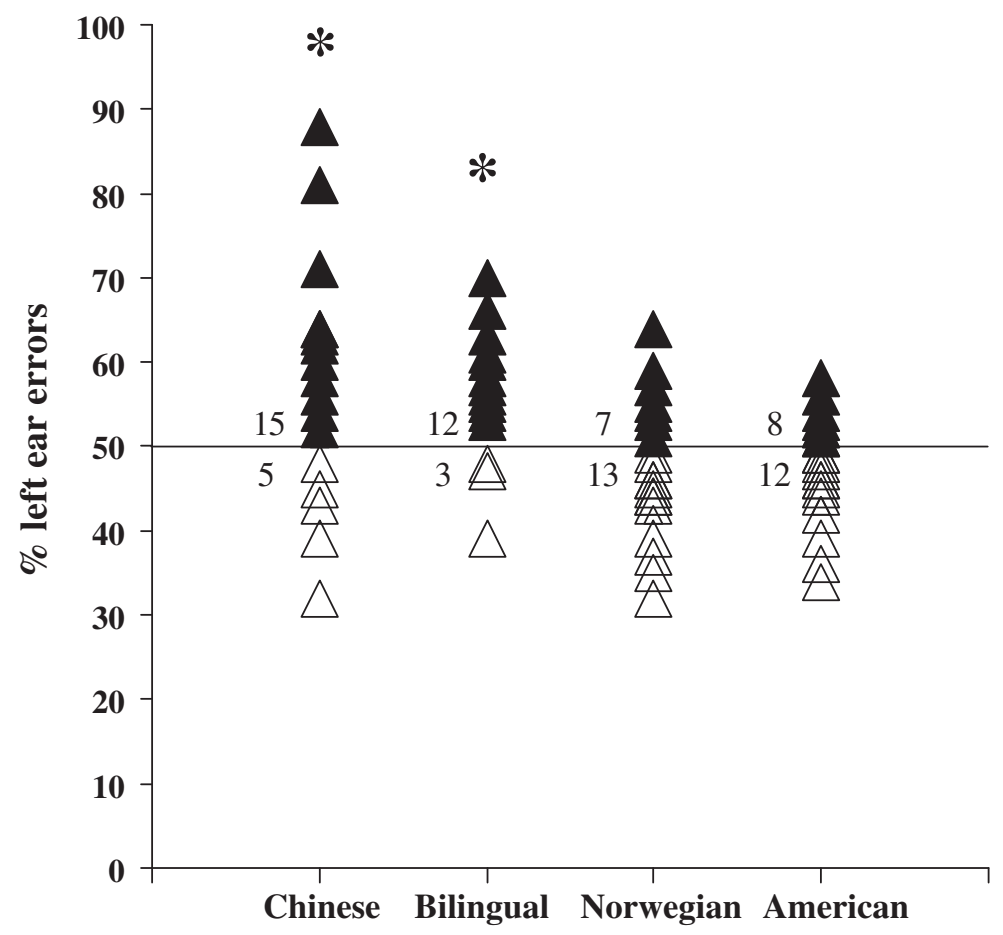

Figure 2. The number of listeners showing $(\Delta)$ REA and $(\triangle)$ LEA for the Chinese $(n=20)$, bilingual $(n=15)$, Norwegian $(n=20)$, and American $(n=20)$ groups. ${ }^{*}$ The difference in the frequency of occurrence of REA and LEA is significant at $p<.05$.

Table 2. Mean number of errors for the left and right ears for each of the four individual tones (Tone 1, Tone 2, Tone 3, and Tone 4)

\begin{tabular}{lcccc}
\hline \hline & $\begin{array}{c}\text { Tone 1 } \\
\text { L-R }\end{array}$ & $\begin{array}{c}\text { Tone 2 } \\
\text { L-R }\end{array}$ & $\begin{array}{c}\text { Tone 3 } \\
\text { L-R }\end{array}$ & $\begin{array}{c}\text { Tone 4 } \\
\text { L-R }\end{array}$ \\
\hline Chinese & $6-5$ & $6-5$ & $22-18$ & $5-3$ \\
Bilingual & $6-5$ & $9-9$ & $17-13$ & $9-6$ \\
Norwegian & $7-7$ & $6-8$ & $8-8$ & $12-12$ \\
American & $5-7$ & $9-10$ & $8-7$ & $12-12$ \\
\hline \hline
\end{tabular}

Note: L, left-ear errors; R, right-ear errors.

Norwegian or American listeners. A two-factor repeated measures ANOVA was calculated for each listener group with number of errors as the dependent variable and ear (left, right) and tone $(1,2,3,4)$ as within-subjects factors.

For the native Chinese group, a significant difference for ear was obtained, $F(1,19)=4.98, p<.038$, with more left-ear errors $(37)$ than right-ear errors $(31)$, 
Table 3. Chinese $(n=20)$, bilingual $(n=15)$, Norwegian $(n=20)$, and American $(n=20)$ listeners' evaluation of the dichotic task

\begin{tabular}{|c|c|c|c|c|c|c|c|c|c|c|c|}
\hline & \multirow[b]{2}{*}{ Language $^{a}$} & \multicolumn{2}{|c|}{ Better Ear } & \multicolumn{4}{|c|}{ Easiest Tone } & \multicolumn{4}{|c|}{ Most Difficult Tone } \\
\hline & & $\mathrm{L}$ & $\mathrm{R}$ & 1 & 2 & 3 & 4 & 1 & 2 & 3 & 4 \\
\hline Chinese & 17 & 6 & 9 & 4 & 2 & 0 & 11 & 3 & 2 & 12 & 0 \\
\hline Bilingual & 12 & 2 & 9 & 7 & 1 & 0 & 7 & 2 & 5 & 8 & 0 \\
\hline Norwegian & 15 & 12 & 4 & 8 & 2 & 6 & 3 & 3 & 8 & 2 & 7 \\
\hline American & 20 & 10 & 3 & 8 & 0 & 12 & 0 & 4 & 7 & 2 & 7 \\
\hline
\end{tabular}

Note: The number in each cell represents the number of listener responses.

${ }^{a}$ The stimuli were language-like; L, left; R, right.

revealing a significant REA. A significant main effect of tone was also observed, $F(3,57)=42.51, p=.000$, with post hoc analyses (Tukey HSD) indicating that, across ears, the number of errors for Tone 3 (40 errors) was significantly greater than for the other three tones (Tone 1, 11 errors; Tone 2, 11 errors; Tone 4, 8 errors). In addition, the interaction of ear and tone was also significant, $F(3,58)=$ $3.33, p<.019$, suggesting that the magnitude of REA is greater for Tone 3 than for the other three tones. Consistent with the native patterns, the bilingual listeners revealed a reliable difference for ear, $F(1,14)=7.12, p<.018$, indicating a significant REA, with more left-ear errors (44) than right-ear errors (33). There was also a significant main effect of tone, $F(3,42)=7.99, p=.000$, with post hoc analyses (Tukey HSD) revealing that the number of errors for Tone 3 (30) was significantly greater than for the other three tones (Tone 1, 11; Tone 2, 18; Tone 4, 15 errors). Likewise, the interaction of ear and tone was significant, $F(3,42)=$ $4.69, p<.007$, suggesting that the magnitude of REA is greater for Tone 3 than for the other three tones.

No significant main effect for ear was observed for the Norwegian listeners, $F(1,19)=1.8, p>.196$, although the effect of tone reached significance, $F(3,57)=6.27, p<.001$. Post hoc analyses (Tukey HSD) showed that for both ears, the number of errors for Tone 4 ( 24 errors) was significantly greater than for Tone 1 ( 14 errors), Tone 2 (14 errors), and Tone 3 (16 errors). Similarly, the American listeners showed no significant difference for ear, $F(1,19)=3.08, p>.096$, but there was a significant main effect of tone, $F(3,57)=9.18, p=.000$, with post hoc analyses (Tukey HSD) showing that the number of errors for Tone 4 (24 errors) was significantly greater than for Tone 1 (12 errors ) and Tone 3 (15 errors).

\section{Listeners' self-evaluation}

After the dichotic test, all listeners were asked four questions: were the stimuli language-like, which ear could better identify the tones, which tones were the easiest to identify, and which tones were the most difficult to identify. Table 3 lists the listeners' responses. 
First, in every group, most of the listeners considered the stimuli language-like. It is interesting to note that, although none of the American listeners had any previous experience with Chinese or any tone language, they all still considered the stimuli language-like. Second, in agreement with the behavioral data showing a significant REA, more Chinese and bilingual listeners considered their right ear better at identifying the tones. By contrast, Norwegian and American listeners overwhelmingly claimed that their left ear was the better ear for identifying the tones, although no significant LEA was revealed. Third, listeners' impression of tone difficulty was also highly consistent with the behavioral data; Chinese and bilingual listeners found Tone 3 to be most difficult, and Tone 4 the easiest, whereas American and Norwegian listeners found Tones 2 and 4 to be most difficult and Tones 1 and 3 the easiest. These evaluative ratings are consistent with the behavioral data showing the Chinese and bilingual listeners made most errors on Tone 3 whereas the Norwegian and American listeners made the most errors on Tone 4. In general, then, it seems that listeners' self-evaluation authentically reflected their actual performance.

\section{DISCUSSION}

The results of the present study revealed different patterns in the processing of Mandarin tones by native and nonnative listeners. For the Chinese listeners, errors made with the left ear exceeded those with the right ear, demonstrating a significant left-hemisphere advantage (an REA) for the processing of Mandarin tones by native Chinese speakers. Similar to the pattern shown by the Chinese listeners, the English-Mandarin bilinguals also exhibited a left-hemisphere (an REA) superiority. In contrast, no ear advantage in processing Mandarin tone was found for the Norwegian listeners, despite their experience with tonal contrasts in Norwegian. Likewise, for the American English listeners without any tone background, there was no ear preference. These results are in agreement with previous dichotic listening studies for other tone languages, with aphasia studies, and with imaging studies (e.g., Gandour et al., 2000; Ryalls \& Reinvang, 1986; Van Lancker \& Fromkin, 1973), suggesting that tones are predominantly lateralized in the left hemisphere when they are processed as distinctive linguistic features in a particular language.

\section{Chinese listeners}

The degree of laterality for the Mandarin listeners, a 57\% POE, is highly comparable to that for the Thai listeners in the Van Lancker and Fromkin (1973) study, in which $57.3 \%$ of the errors occurred in the left ear. Moreover, this right ear advantage for processing tone occurred for most listeners. In the current study, 15 out of 20 Mandarin listeners exhibited an REA (corresponding to $75 \%$ of the sample), which was similar to Van Lancker and Fromkin's (1973) 16 out of 21 (76\%). To a lesser degree, in Moen's (1993) study on Norwegian, the right ear was reported more correctly than the left ear by 21 out of 32 listeners $(66 \%)$ in a first task and by 12 out of 23 listeners (52\%) in a second task. The present results provide 
converging evidence that for native listeners the perception of tonal contrasts in Mandarin Chinese, similar to other tone languages like Thai and Norwegian, is largely a property of the left hemisphere.

Thus, it appears that native speakers do process lexical tone depending on whether pitch is used to convey analytic linguistic information, as the functional hypothesis suggests. To account for differences in lateralization between the perception of linguistic tone and other pitch-related contrasts, Van Lancker (1980) proposed a hypothetical continuum of hemispheric specialization of pitch contrasts from the most linguistic use of pitch associated with the left hemisphere to the least linguistic use of pitch associated with right hemisphere specialization. Hence, the most highly structured level of pitch pattern, phonological tone, falls at one end of the scale whereas emotional and personal patterning of pitch phenomena are at the other end of the scale. In between these two extremes are pitch contrasts at the lexical and syntactic levels, such as stress and intonation. Previous research supports this functional hypothesis, finding a left-hemisphere superiority for the processing of lexical stress (e.g., hotdog, hot dog in English; Baum \& Pell, 1999; Emmorey, 1987), whereas more right-hemisphere dominance is found in the processing of linguistic intonation (Blumstein \& Cooper, 1974) and even greater degree of right-hemisphere dominance for affective prosody (Buchanan et al., 2000). The present findings support the hypothesis that, because Mandarin tones are highly structured phonologically and are specified in the lexicon, they are processed in the left hemisphere by native Chinese listeners.

Despite the present finding of left hemisphere superiority in the processing of Mandarin tones, some participation of the right hemisphere is also possible, as the degree of REA varied across participants. It should be noted that the lateralization of the brain is a tendency, in that "dominant" does not exclude activity in the other hemisphere. Even though the left hemisphere plays a dominant role in the linguistic realm, the right hemisphere is also involved in the processing of language (Berry et al., 1996; Carrol, 1994; Chiarello, 1991). Nevertheless, the native Chinese listeners predominantly processed lexical tone in the left hemisphere, in accordance with the processing of language in general.

\section{English-Mandarin bilingual listeners}

The present results show that tone processing for the English-Mandarin bilinguals authentically follows the patterns exhibited by the native Mandarin listeners. The degree of left-hemisphere superiority for the bilinguals (POE: 56\%) is similar to that for the Chinese listeners (POE: 57\%). In terms of frequency, $80 \%$ of the bilingual listeners exhibited left-hemisphere dominance, which is comparable with the Chinese group (75\%). Thus, it seems that the proficient bilinguals do not differ from native Chinese speakers in terms of left-hemisphere dominance for processing Mandarin tones. Because the bilinguals in the present study were highly proficient Mandarin speakers, it could then be claimed that when bilinguals achieve nearnative fluency in Mandarin, their tone processing patterns also become nativelike. 
Although there is little research on the bilingual processing of lexical tone, the current results appear consistent with previous findings of bilingual language processing in general. For example, Soares (1984) shows equal levels of lefthemisphere dominance in the processing of English by native speakers of Portuguese who were also highly proficient in English (Portuguese-English bilinguals), compared to the English monolingual controls. Together these results suggest that highly proficient bilinguals can achieve a nativelike processing pattern in the L2.

Concerning age at target language acquisition, previous studies have shown that early (rather than late) bilinguals demonstrated left-hemisphere dominance for the target language (Sussman et al., 1982; Wuillemin \& Richardson, 1994). The present data support these findings, with the English-Mandarin bilinguals who acquired Mandarin at an early age processing Mandarin tones primarily in the left hemisphere. However, because the English-Mandarin listeners were proficient, as well as early, bilinguals, it is difficult to determine whether their Mandarin tone processing pattern is due to acquired proficiency, age of acquisition, or both. Although proficiency and age are usually confounded, previous research indicates that attained proficiency may be more important than age of acquisition as a determinant of the processing of L2 (Evans, Workman, Mayer, \& Crowley, 2002; Perani et al., 1998).

Some studies have found different patterns for bilingual processing of the target language, ranging from equally contributing "interhemispheric" processing (e.g., $\mathrm{Ke}, 1992$ ) to greater right-hemispheric involvement (e.g., Mildner, 1999). However, these studies are not directly comparable with the current study, because the bilinguals involved in these studies were less proficient in the respective target language. For example, the English-Chinese bilinguals in the study by Ke (1992) were third-year Chinese learners; and the Croatian-English bilinguals in Mildner (1999) had learned English for 8 years. In these cases, lack of proficiency may account for the finding of less left-hemispheric involvement. In a recent neuroimaging study also using elementary learners, results showed that improved proficiency as a result of intense Mandarin tone training is manifested by increased brain activity in both hemispheres (Wang et al., 2003), including an increase in the spatial extent of activation and an emergence of additional areas of activation. Using more proficient learners, Albanese (1985) showed that growing target language proficiency increases right hemisphere participation for English-French bilinguals, whereas Schouten et al. (1985) found comparable levels of left hemisphere participation for the target language for proficient compared to nonproficient Dutch-English bilinguals. Although these studies involve bilinguals with different proficiency levels and are thus not directly comparable, it is worth noting that they are consistent in that an overall left hemisphere advantage does exist for all the bilinguals, regardless of the extent of right hemisphere participation.

In sum, for the highly proficient bilinguals in the current study, Mandarin tone is predominantly lateralized to the left hemisphere, similar to the processing of other distinctive linguistic features by native listeners. Although the degree of right hemisphere involvement and approximation to the native pattern does depend on the listener's proficiency in the target language, for these bilinguals who learned 
Mandarin from an early age and show proficiency in the language, tone is primarily processed in the left hemisphere.

\section{Norwegian listeners}

Unlike the native speakers of Mandarin who demonstrate an REA, the Norwegian listeners did not show any significant ear preference for Mandarin tone processing. Although the Norwegian listeners had no knowledge of the Mandarin tones, they did have experience with tonal distinctions in their native language. Previous research has found that the Norwegian tones are primarily processed in the left hemisphere for native speakers of Norwegian (Moen, 1993). However, the results of the current study show that the left hemisphere superiority for Norwegian tone processing did not carry over to the processing of Mandarin tones by Norwegian listeners. Thus, it appears that familiarity with the pitch distinctions in Norwegian did not lead to similar patterns when processing Mandarin. This is probably due to the contrastive pitch patterns specific to Mandarin not being linguistically meaningful for the Norwegian listeners. In addition, as mentioned earlier, because the realization of tone in Norwegian is dependent on stress, the functional load of Norwegian tone is relatively low compared to that in Mandarin (Kristoffersen, 2000), making the Chinese tones more distant from their experience with Norwegian tones.

Behavioral studies with phonetic segments have shown analogous results. For example, using the dichotic listening paradigm, Best and Avery (1999) investigated Zulu versus English listeners' hemispheric processing for Zulu clicks. They found that only Zulu listeners showed a right ear (left hemisphere) advantage, whereas the English listeners did not show any ear preference. Although the perception of consonants is generally found to be lateralized in the left hemisphere (Shankweiler \& Studdert-Kennedy, 1967; Zatorre et al., 1992), familiarity with the properties of consonants in general (e.g., formant transitions) for the English listeners did not lead to a left hemisphere advantage for the processing of Zulu click consonants. The present results from the dichotic listening task also align well with the findings from imaging studies. For example, neuroimaging studies examining the brain activation patterns in the processing of Thai tones revealed significant activation in the lefthemisphere language-sensitive regions for tone processing by native Thai speakers but not for Chinese speakers, despite Chinese speakers' familiarity with lexical tone in Mandarin (Gandour et al., 2000, 2002). These results are consistent with the present findings for the Norwegian listeners. Together, they seem to indicate a language-dependent lateralization effect. That is, the left hemisphere specification seems to occur only when the information is linguistically significant for the listeners.

\section{American listeners}

The present study found no ear advantage in the processing of Mandarin tones by the American listeners. These data are in agreement with studies showing no ear advantage in the dichotic listening of Thai tones by English listeners (Van Lancker 
\& Fromkin, 1973), and neuroimaging results revealing a lack of left hemisphere engagement in the processing of Thai (Gandour et al., 2000) and Mandarin (Klein et al., 2001) tones by English listeners. Given these findings, it would appear that left hemisphere lateralization of lexical tone is not a characteristic of nonnative speakers with no tone background.

It should also be noted that the American (as well as Norwegian) listeners did not show a left-ear (right-hemisphere) preference for the tones either. These listeners might have treated the Mandarin tones as language after all. (Recall that they all claimed to have heard the stimuli as language, as shown in Table 3.) Although they have no prior experience with Mandarin tone, their experience with the processing of lexical stress (Baum \& Pell, 1999; Emmorey, 1987) suggests that left hemisphere processing of prosodic features is not completely novel to them. As a consequence, both of their hemispheres were involved in the processing of the Mandarin tones.

\section{CONCLUSIONS}

Taken together, the present results examining Mandarin tone processing by four groups of listeners with different linguistic backgrounds (Chinese, EnglishMandarin bilinguals, Norwegian, and American) provide consistent support for the functional hypothesis (Van Lancker, 1980). Chinese listeners show an REA in the dichotic perception of Mandarin tones. This is in general agreement with previous research on other tone languages, demonstrating a lefthemisphere superiority in the processing of lexical tone by native tone-language speakers.

In contrast, nonnative processing of Mandarin tones produced different lateralization patterns. For both the American English listeners without any experience with Mandarin or other tone languages and for the Norwegian listeners who have experience with Norwegian but not with Mandarin tones, no hemisphere advantage was observed. These data indicate bilateral processing of Mandarin tones for nonnative listeners naive to Mandarin, regardless of their prior knowledge of tone from their native language. For the American listeners, the phonological function and the lexical specification of tone were novel. Although the Norwegian listeners were familiar with phonemic pitch distinctions at the lexical level, the pitch patterns specifically associated with Mandarin tones were not phonologically and lexically significant. Consequently, both the American and Norwegian listeners were unlikely to process Mandarin tones predominantly in the left hemisphere, as the native Chinese listeners did.

Different from the Norwegian and American listeners, the English-Mandarin bilinguals' tone processing followed the same pattern as the Chinese listeners, exhibiting significant left-hemisphere dominance. It seems that for these proficient early bilinguals, who have acquired the functional use of the Mandarin pitch contrasts, hemispheric processing of Mandarin tones becomes nativelike. These results indicate a dynamic pattern in the processing of Mandarin tones by nonnative speakers, from no hemispheric lateralization to nativelike left-hemisphere dominance. 


\section{APPENDIX A}

The 16 target stimuli (4 syllables $\times 4$ tones)

\begin{tabular}{lcl}
\hline \hline Pinyin & Tone & English Gloss $^{a}$ \\
\hline fan1 & 1 & Sail \\
fan2 & 2 & Annoy \\
fan3 & 3 & Reverse \\
fan4 & 4 & Meal \\
guol & 1 & Pan \\
guo2 & 2 & Country \\
guo3 & 3 & Fruit \\
guo4 & 4 & Pass \\
huil & 1 & Gray \\
hui2 & 2 & Return \\
hui3 & 3 & Destroy \\
hui4 & 4 & Meeting \\
shil & 1 & Teacher \\
shi2 & 2 & Ten \\
shi3 & 3 & History \\
shi4 & 4 & Right \\
\hline \hline
\end{tabular}

${ }^{a}$ The most common meaning is used here.

\section{NOTES}

1. In addition to tone, different terms have been used to refer to the Norwegian tonal contrasts, including toneme, tonal accent, and pitch accent (Kristoffersen, 2000).

2. Data from the native Chinese and American English listeners have previously been reported in Wang et al. (2001).

\section{REFERENCES}

Albanese, J. F. (1985). Language lateralization in English-French bilinguals. Brain and Language, 24, 284-296.

Baum, S. R., \& Pell, M. D. (1999). The neural bases of prosody: Insights from lesion studies and neuroimaging. Aphasiology, 13, 581-608.

Berry, I., Manelfe, C., Mueller, E., Franconi, J. M., Boulanouar, K., Demonet, J. F., Chollet, F., Rascol, O., \& Clanet, M. (1996). Functional magnetic resonance of motor and verbal tasks. In L. Heuser \& M. Oudkerk (Eds.), Advances in MRI (pp. 27-33). Berlin: Blackwell Science.

Best, C. T., \& Avery, R. A. (1999). Left-hemisphere advantage for click consonants is determined by linguistic significance and experience. Psychological Science, 10, 65-70.

Blumstein, S., \& Cooper, W. (1974). Hemispheric processing of intonation contours. Cortex, 10, 146158.

Bryden, M. P. (1988). An overview of the dichotic listening procedure and its relation to cerebral organization. In K. Hugdahl (Ed.), Handbook of dichotic listening: Theory, methods and research. Chichester, UK: Wiley.

Bryden, M. P., \& Murray, J. E. (1985). Toward a model of dichotic listening performance. Brain and Cognition, 4, 241-257. 
Buchanan, T., Lutz, K., Mirzazade, S., Specht, K., Shah, N., Zilles, K., \& Jäncke, L. (2000). Recognition of emotional prosody and verbal components of spoken language: An fMRI study. Cognitive Brain Research, 9, 227-238.

Carrol, D. W. (1994). Psychology of language. Pacific Grove, CA: Brooks/Cole.

Chiarello, C. (1991). Interaction of word meanings by the cerebral hemisphere: One is not enough. In P. J. Schwanenflugel (Ed.), The psychology of word meaning (pp. 251-278). Hillsdale, NJ: Erlbaum.

Curry, F. K. W. (1967). A comparison of left-handed and right-handed subjects on verbal and nonverbal dichotic listening tasks. Cortex, 3, 343-352.

Cutting, J. E. (1974). Two left-hemisphere mechanisms in speech perception. Perception and Psychophysics, 16, 601-612.

Dwyer, J., Blumstein, S. E., \& Ryalls, J. (1982). The role of duration and rapid temporal processing on the lateral perception of consonants and vowels. Brain and Language, 17, 272-286.

Emmorey, K. (1987). The neurological substrates for prosodic aspects of speech. Brain and Language, 30, 305-320.

Evans, J., Workman, L., Mayer, P., \& Crowley, K. (2002). Differential bilingual laterality: Mythical monster found in Wales. Brain and Language, 83, 291-299.

Fintoft, K. (1970). Acoustical analysis and perception of tonemes in some Norwegian dialects. Oslo: Universitetsforlaget.

Gandour, J., \& Dardarananda, R. (1983). Identification of tonal contrasts in Thai aphasic patients. Brain and Language, 18, 98-114.

Gandour, J., Petty, S. H., \& Dardarananda, R. (1988). Perception and production of tone in aphasia. Brain and Language, 35, 201-240.

Gandour, J., Dzemidzic, M., Wong, D., Lowe, M., Tong, Y., Hsieh, L, Satthamnuwong, N., \& Lurito, J. (2003). Temporal integration of speech prosody is shaped by language experience: An fMRI study. Brain and Language, 84, 318-336.

Gandour, J., Wong, D., Hsieh, L., Weinzapfel, B., Van Lancker, D., \& Hutchins, G. D. (2000). A crosslinguistic PET study of tone perception. Journal of Cognitive Neuroscience, 12, 207222.

Gandour, J., Wong, D., Lowe, M., Dzemidzic, M., Satthamnuwong, N., Tong, Y., \& Li, X. (2002). A cross-linguistic fMRI study of spectral and temporal cues underlying phonological processing. Journal of Cognitive Neuroscience, 14, 1076-1087.

Goodglass, H., \& Calderon, M. (1977). Parallel processing of verbal and musical stimuli in right and left hemispheres. Neuropsychologia, 15, 397.

Ke, C. (1992). Dichotic listening with Chinese and English tasks. Journal of P sycholinguistic Research, $21,463-471$.

Kimura, D. (1964). Left-right differences in the perception of melodies. Quarterly Journal of Experimental Psychology, 16, 335-358.

Kimura, D. (1967). Functional asymmetry of the brain in dichotic listening. Cortex, 3, 163-168.

Klein, D., Zatorre, R. J., Milner, B., \& Zhao, V. (2001). A cross-linguistic PET study of tone perception in Mandarin Chinese and English speakers. Neuroimage, 13, 646-653.

Kristoffersen, G. (2000). The phonology of Norwegian. Oxford: Oxford University Press.

Lehiste, I. (1970). Suprasegmentals. Cambridge, MA: MIT Press.

Mazzucchi, A., Parma, M., \& Cattelani, R. (1981). Hemispheric dominance in the perception of tonal sequences in relation to sex, musical competence and handedness. Cortex, 17, 291302.

Mertus, J. (1989). BLISS manual. Brown University, Providence, RI.

Mildner, V. (1999). Functional cerebral asymmetry for verbal stimuli in a foreign language. Brain and Cognition, 40, 197-201.

Moen, I. (1993). Functional lateralization of the perception of Norwegian word tones-Evidence from a dichotic listening experiment. Brain and Language, 44, 400-413.

Naeser, M. A., \& Chan, S. W. C. (1980). Case study of a Chinese aphasic with the Boston diagnostic aphasia exam. Neuropsychologia, 18, 389-410.

Oldfield, R. C. (1971). The assessment and analysis of handedness: The Edinbourgh inventory. Neuropsychologia , 9, 97-113.

Packard, J. L. (1986). Tone production deficits in nonfluent aphasic Chinese speech. Brain and Language, 29, 212-223. 
Perani, D., Paulesu, E., Sebastian Galles, N., Dupoux, E., Dehaene, S., Bettinardi, V., Cappa, S. F., Fazio, F., \& Mehler, J. (1998). The bilingual brain: Proficiency and age of acquisition of the second language. Brain, 121, 1841-1852.

Robin, D., Tranel, D., \& Damasio, H. (1990). Auditory perception of temporal and spectral events in patients with focal left and right cerebral lesions. Brain and Language, 39, 539-555.

Ryalls, J., \& Reinvang, I. (1986). Functional lateralization of linguistic tones: Acoustic evidence from Norwegian. Language and Speech, 29, 389-398.

Schouten, M. E. H., van Dalen, T. E., \& Klein, A. J. J. (1985). Ear advantage and second language proficiency. Journal of Phonetics, 13, 53-60.

Shankweiler, D., \& Studdert-Kennedy, M. (1967). Identification of consonants and vowels presented to left and right ears. Quarterly Journal of Experimental Psychology, 19, 59-63.

Soares, C. (1982). Converging evidence for left hemisphere language lateralization in bilinguals. Neuropsychologia, 20, 653-659.

Soares, C. (1984). Left-hemisphere language lateralization in bilinguals: Use of the concurrent activities paradigm. Brain and Language, 23, 86-96.

Soares, C., \& Grosjean, F. (1981). Left hemisphere language lateralization in bilinguals and monolinguals. Perception and Psychophysics, 29, 599-604.

Studdert-Kennedy, M., \& Shankweiler, D. (1970). Hemispheric specialization for speech perception. Journal of the Acoustical Society of America, 48, 579-594.

Sussman, H. M., Franklin, P., \& Simon, T. (1982). Bilingual speech: Bilingual control? Brain and Language, $15,125-142$.

Van Lancker, D. (1980). Cerebral lateralization of pitch cues in the linguistic signal. Papers in Linguistics: International Journal of Human Communication, 13, 201-277.

Van Lancker, D., \& Fromkin, V. A. (1973). Hemispheric specialization for pitch and "tone”: Evidence from Thai. Journal of Phonetics, 1, 101-109.

Van Lancker, D., \& Fromkin, V. A. (1978). Cerebral dominance for pitch contrasts in tone language speakers and in musically untrained and trained English speakers. Journal of Phonetics, 6, $19-23$.

Wang, Y., Jongman, A., \& Sereno, J. A. (2001). Dichotic perception of Mandarin tones by Chinese and American listeners. Brain and Language, 78, 332-348.

Wang, Y., Sereno, J.A., Jongman, A., \& Hirsch, J. (2003). fMRI evidence for cortical modification during learning of Mandarin lexical tone. Journal of Cognitive Neuroscience, 15, 1019-1027.

Wong, P. C. M. (2002). Hemispheric specialization of linguistic pitch patterns. Brain Research Bulletin, $59,83-95$.

Wuillemin, D., \& Richardson, B. (1994). Right hemisphere involvement in processing later-learned languages in multilinguals. Brain and Language, 46, 620-636.

Zatorre, R. J., Evans, A. C., Meyer, E., \& Gjedde, A. (1992). Lateralization of phonetic and pitch discrimination in speech processing. Science, 256, 846-849. 